\title{
Evaluating high risks in large-scale projects using an extended VIKOR method under a fuzzy environment
}

\author{
S. Ebrahimnejad ${ }^{\mathrm{a}}$, S. M. Mousavi ${ }^{\mathrm{b}}$, R. Tavakkoli-Moghaddam ${ }^{\mathrm{c}}$ and M. Heydar ${ }^{\mathrm{d}}$
}

${ }^{a}$ Department of Industrial Engineering, Islamic Azad University, Karaj Branch, Karaj, Iran

${ }^{b}$ Young Researches Club, South Tehran Branch, Islamic Azad University, Tehran, Iran

${ }^{c}$ Department of Industrial Engineering, College of Engineering, University of Tehran, Tehran, Iran

${ }^{d}$ Department of Industrial and Manufacturing Engineering, University of Wisconsin, Milwaukee, USA

\begin{tabular}{|c|c|}
\hline ARTICLEINFO & ABSTRACT \\
\hline $\begin{array}{l}\text { Article history: } \\
\text { Received 25 October } 2011 \\
\text { Received in revised form } \\
\text { November, 2, 2011 } \\
\text { Accepted December, } 12011 \\
\text { Available online } \\
\text { 4 December 2011 } \\
\text { Keywords: } \\
\text { Risk evaluation } \\
\text { Large-scale projects } \\
\text { Fuzzy sets } \\
\text { VIKOR }\end{array}$ & $\begin{array}{l}\text { The complexity of large-scale projects has led to numerous risks in their life cycle. } \\
\text { This paper presents a new risk evaluation approach in order to rank the high risks in } \\
\text { large-scale projects and improve the performance of these projects. It is based on the } \\
\text { fuzzy set theory that is an effective tool to handle uncertainty. It is also based on an } \\
\text { extended VIKOR method that is one of the well-known multiple criteria decision- } \\
\text { making (MCDM) methods. The proposed decision-making approach integrates } \\
\text { knowledge and experience acquired from professional experts, since they perform the } \\
\text { risk identification and also the subjective judgments of the performance rating for } \\
\text { high risks in terms of conflicting criteria, including probability, impact, quickness of } \\
\text { reaction toward risk, event measure quantity and event capability criteria. The most } \\
\text { notable difference of the proposed VIKOR method with its traditional version is just } \\
\text { the use of fuzzy decision-matrix data to calculate the ranking index without the need } \\
\text { to ask the experts. Finally, the proposed approach is illustrated with a real-case study } \\
\text { in an Iranian power plant project, and the associated results are compared with two } \\
\text { well-known decision-making methods under a fuzzy environment. }\end{array}$ \\
\hline
\end{tabular}

\section{Introduction}

Risk management can be helpful in large-scale projects if it is performed in a systematic manner from planning phase through the project completion. An unsystematic and arbitrary risk management can highly affect the success of these projects since most of the identified risks are dynamic throughout the project life cycle (Nieto-Morote \& Ruz-Vila, 2011). In general, project risk management contains a four-phase process (Ebrahimnejad et al., 2008; Makui et al., 2010; Mousavi et al., 2011a; Mousavi et al. 2011b; Nieto-Morote, \& Ruz-Vila, 2011):

- Risk identification: The process of obtaining which risks may have impacts on the project, and documenting their features.

\footnotetext{
* Corresponding author Cell phone: +98 2614182333

E-mail: ibrahimnejad@kiau.ac.ir (S. Ebrahimnejad)

(c) 2012 Growing Science Ltd. All rights reserved. doi: 10.5267/j.ijiec.2011.12.001
} 
- Risk evaluation: The process of prioritizing risks for further analysis by evaluating their probability of occurrence and impact.

- Risk response: The process of developing alternatives and responses in order to increase opportunities and to reduce threats to the project objectives.

- Risk monitoring: The process of performing a risk response plan, tracking identified risks, monitoring residual risks, identifying new risks and analyzing the risk process effectiveness throughout the project.

Evaluating the high risks of large-scale projects is a multiple criteria decision making (MCDM) problem with multiple qualitative and quantitative criteria. The MCDM provides an effective framework for the project management team based on evaluation of multiple and often conflicting criteria. Compromise solution methods are regarded as important types of the MCDM and are wide spread in real-life decision situations. There are several methods with different characteristics and constraints. Hence, it is not possible to apply them in any decision-making problem (Ebrahimnejad et al., 2010). On the other hands, in an uncertain large-scale project environment, the evaluation process of high risks is not often performed sufficiently and exactly, because the available data and information are vague, inexact, imprecise and uncertain by nature of these projects. The decisionmaking process dealing with the evaluating risks should be based on uncertain and ill-defined information. To solve the vagueness, ambiguity and subjectivity of experts or decision maker (DM)' judgment, the fuzzy set theory was introduced to express the linguistic terms in this process (Ebrahimnejad et al., 2009).

The concept of a linguistic variable is very useful in dealing with situations, which are too complex or not well defined to be reasonably described in conventional quantitative expressions (Kaufmann, A., \& Gupta, 1985; Zimmermann, 1991). The concept of combining the fuzzy set theory and MCDM is referred to as fuzzy MCDM. This approach helps DMs solve complex decision-making problems in a systematic, consistent, effective and productive way. It has been widely applied to tackle DM problems with multiple criteria and alternatives (Kahraman et al., 2007; Tsuar et al, 2002; Wang \& Chang, 2007).

Previous studies illustrated some MCDM and fuzzy MCDM methods for identifying and evaluating the risks of large-scale projects. Mustafa and Al-Bahar (1991) proposed the analytic hierarchy process (AHP) and Dey et al. (1994) considered both a subjective and objective approach to risk evaluation based on experts' judgments. However, their approaches fail to combine risk evaluation with the project management processes. Dey (2002) designed a combined AHP and decision tree approach to handle the risks that quantifies both probability and impact of risk. This framework considered both time and cost parameters of projects. However, it cannot be used in evaluating project level risk for choosing least risky project.

More recently, Dey and Ogunlana (2004) used risk management for managing build-operate-transfer (BOT) projects and provided a framework for choosing most suitable risk management method. However, this does not assist much to manage risk across each level of projects. Zayed et al. (2008) used the AHP for evaluating risk in Chinese highway projects. Chin et al. (2008) considered an evidential reasoning-AHP system for the NPD project screening model. These frameworks rank risk factors and rank alternative projects. However, their approaches do not discuss on managing projects effectively using risk management methodology. Zeng et al. (2007) proposed a risk assessment model based on modified AHP and fuzzy reasoning to deal with the uncertainties arising in the construction projects. Makui et al. (2010) presented a methodology for identifying and evaluating risks of largescale projects concurrently by applying a fuzzy multi-attribute group decision-making approach.

Ebrahimnejad et al. (2009) introduced some effective criteria and attributes for evaluating risks, and presented a model for risk evaluation in construction projects based on fuzzy MCDM. Furthermore, 
Ebrahimnejad et al. (2010) identified common risks in BOT projects. Then, a fuzzy technique for order preference by similarity to ideal solution (TOPSIS) and fuzzy linear programming technique for multidimensional analysis of preference (LINMAP) methods were proposed to rank higher risks in the large-scale projects. Nieto-Morote and Ruz-Vila (2011) proposed a risk evaluation methodology based on the fuzzy sets theory and on the AHP, which was used to structure a large number of risks. Kumar Dey (2010) developed a combined approach based on the AHP and risk map to be used in complex projects and helps manage risk throughout the project life cycle.

Based on the studies explained, it can be seen that it is very important to develop a new project risk evaluation approach in order to analyze the associated risks involved in these projects, particularly in developing countries due to various kinds of uncertainties. Effective resource planning is an essential manner for each project organization with limited resources, and the risk importance from the professional experts should be evaluated, precisely.

The purpose of this paper is to present a suitable fuzzy compromising solution approach based on an extended VIKOR method for evaluating high risks in the large-scale projects. VIKOR stands for the Serbian name, "vlsekriterijumska optimizacija i kompromisno resenje", which means multi-criteria optimization and compromise solution. The idea behind this is to provide the necessary action for responding to the risks occurred in the project risk management process. Finally, an application of the proposed approach is studied for a real-world large-scale project of a power industry. Furthermore, two powerful MCDM methods, namely TOPSIS, and LINMAP, are applied in a fuzzy environment; their results are compared with the proposed VIKOR approach and then its merits are highlighted.

The rest of this paper is organized as follows. In Sections 2 and 3, compromise solution method and some basic concepts on fuzzy sets are briefly introduced, respectively. In Section 4, the fuzzy VIKOR method is developed to evaluate high risks in large-scale projects. Section 5 provides the background information for the case study problem in order to illustrate their potential applications in large-scale projects. In Section 6, the computational results are discussed and the proposed approach is compared with two compromise solution methods. Finally, conclusions are provided in Section 7.

\section{VIKOR Method}

MCDM methods are major parts of the decision theory and analysis. The aim of compromise solution methods as the well-known category of the MCDM is to help DMs learn about the problems. These methods are powerful tools widely used for evaluation problems containing multiple and are wide spread in real-life decision situations (Al-Subhi Al-Harbi, 2001; Büyüközkan \& Ruan, 2008; Ebrahimnejad et al., 2010; Mojtahedi, 2010; Wu et al., 2009). Moreover, compromise solution methods are useful in different circumstances, which cannot be evaluated by the measurement of a simple and single dimension.

Opricovic and Tzeng (2004) are believed to the first who developed the VIKOR method. This method is on the basis of the compromise solution approach. We assume that each alternative is evaluated based on a separate criterion function; the compromise ranking can be achieved by comparing the measure of closeness to the ideal alternative. The multi-criteria measure for the compromise ranking is extended from the $L_{P}$-metric utilized as an aggregating function for a compromise programming method (Opricovic, 1998; Opricovic \& Tzeng, 2007).

Assuming that each alternative is assessed versus each criterion function, the compromise ranking can be performed by comparing the measure of closeness to the ideal alternative. The $m$ alternatives are denoted as $A_{1}, A_{2}, \ldots, A_{m}$. The rating of the $j$ th aspect is denoted by $f_{i j}$, i.e., $f_{i j}$ is the value of $j$ th criterion function for the alternative $A_{i} ; n$ is the number of criteria. Extension of the VIKOR method is started with the following form of $L_{P}$-metric: 
$L_{p i}=\left\{\sum_{j=1}^{n}\left[\left(f_{j}^{*}-f_{i j}\right) /\left(f_{j}^{*}-f_{j}^{-}\right)\right]^{p}\right\}^{1 / p} \quad 1 \leq p \leq \infty ; \quad i=1,2, \ldots, m$.

In VIKOR method, $L_{1 . i}$ (as $S_{i}$ ) and $L_{\infty, i}$ (as $R_{i}$ ) are utilized to formulate a ranking measure. The solution given by $\min S_{i}$ is with a maximum group utility (i.e., majority rule), and the solution provided by $\min R_{i}$ is with a minimum individual regret of the opponent. The compromise ranking algorithm of the VIKOR has the following steps.

(a) Determine the best $f_{j}^{*}$ and the worst $f_{j}^{-}$values of all criterion functions $j=1,2, \ldots, n$. If the $j$ th function represents a benefit, then we have:

$$
\begin{gathered}
f_{i}^{*}=\max _{j} f_{i j} \\
\text { and } \\
f_{i}^{-}=\min _{j} f_{i j}
\end{gathered}
$$

(b) Compute the values $S_{i}$ and $R_{i} ; i=1,2, \ldots, m$, by these relations:

$$
\begin{aligned}
& S_{i}=L_{1, i}=\sum_{j=1}^{m} w_{j}\left(f_{j}^{*}-f_{i j}\right) /\left(f_{j}^{*}-f_{j}^{-}\right), \\
& R_{i}=L_{\infty, i}=\max _{j} w_{j}\left(f_{j}^{*}-f_{i j}\right) /\left(f_{j}^{*}-f_{j}^{-}\right),
\end{aligned}
$$

where $w_{j}$ are the weights of criteria expressing their relative importance.

(c) Compute the values $Q_{i} ; i=1,2, \ldots, m$, by the following relation:

$Q_{i}=v\left(S_{i}-S^{*}\right) /\left(S^{-}-S^{*}\right)+(1-v)\left(R_{i}-R^{*}\right) /\left(R^{-}-R^{*}\right)$

where

$$
\begin{aligned}
& S^{*}=\min _{i} S_{i}, \quad S^{-}=\max _{i} S_{i} \\
& R^{*}=\min _{i} R_{i}, \quad R^{-}=\max _{i} R_{i}
\end{aligned}
$$

$v$ is introduced as weight of the strategy of the majority of criteria or the maximum group utility), here $v=0.5$.

(d) Rank the alternatives, sorting by the values $S, R$ and $Q$ in a non-increasing order.

The VIKOR is a remarkable tool in MCDM, particularly in a situation where the DM is not able, or does not know to describe his/her preference at the beginning of system design. The solution can be accepted by the DMs because it provides a maximum group utility of the majority represented by min $S$ and a minimum of the individual regret of the opponent represented by min $R$. The resulted solutions can be the basis for discussion involving the DM's preference by criteria weights (Opricovic \& Tzeng, 2004; 2007). There are typical applications of VIKOR method in the various industrial fields. For instance, evaluation of software development projects (Büyüközkan \& Ruan, 2008), partners' choice in IS/IT outsourcing projects (Chen \& Wang, 2009), project risk identification and prioritization (Tavakkoli-Moghaddam et al., 2011), and plant location selection (TavakkoliMoghaddam et al., 2011). 


\section{Basic Concept on Fuzzy Sets}

It is often difficult for an expert or DM to assign a precise performance rating to an alternative for the attributes under consideration. The purpose of implementing a fuzzy approach is to assign the relative importance of attributes using fuzzy numbers instead of precise numbers. We briefly review the rationale of fuzzy theory before descriptions of proposed VIKOR method; as follows:

Definition 3.1. A fuzzy set $\tilde{a}$ in a universe of discourse $x$ is characterized by a membership function $\tilde{a}$ which associates with each element $x$ in $X$, a real number in the interval $[0,1]$. The function value $\mu_{\tilde{a}}(x)$ is termed the grade of membership of $x$ in $\tilde{a}$ (Kaufmann \& Gupta, 1985; Zimmermann, 1991). A triangular fuzzy number $\tilde{a}$ can be defined by a triplet $\left(a_{1}, a_{2}, a_{3}\right)$. Its conceptual schema and mathematical form are shown by Eq. (9) (Kaufmann \& Gupta, 1985; Zimmermann, 1991).

$$
\mu_{\tilde{a}}(x)= \begin{cases}0 & ; x \leq a_{1} \\ \frac{x-a_{1}}{a_{2}-a_{1}} & ; a_{1} \leq x \leq a_{2} \\ \frac{a_{3}-x}{a_{3}-a_{2}} & ; a_{2} \leq x \leq a_{3} \\ 0 & ; x \geq a_{3}\end{cases}
$$

Definition 3.2. Let $\tilde{a}=\left(a_{1}, a_{2}, a_{3}\right)$ and $\tilde{b}=\left(b_{1}, b_{2}, b_{3}\right)$ be two triangular fuzzy numbers, then the vertex method is defined to calculate the distance between them, as Eq. (10):

$$
d(\tilde{a}, \tilde{b})=\sqrt{\frac{1}{6}\left[\left(a_{1}-b_{1}\right)^{2}+4\left(a_{2}-b_{2}\right)^{2}+\left(a_{3}-b_{3}\right)^{2}\right]}
$$

Property 3.1. Assuming that both $\tilde{a}=\left(a_{1}, a_{2}, a_{3}\right)$ and $\tilde{b}=\left(b_{1}, b_{2}, b_{3}\right)$ are real numbers, then the distance measurement $d(\tilde{a}, \tilde{b})$ is identical to the Euclidean distance (Xia et al., 2006; Zimmermann, 1991).

Property 3.2. Let $\tilde{a}, \tilde{b}$, and $\tilde{c}$ be three triangular fuzzy numbers. The fuzzy number $\tilde{b}$ is closer to fuzzy number $\tilde{a}$ than the other fuzzy number $\tilde{c}$ if, and only if, $d(\tilde{a}, \tilde{b}) \leq d(\tilde{a}, \tilde{c})$ (Li \& Yang, 2004; 2006; Zimmermann, 1991).

Definition 3.3. Given a triangular fuzzy number $\tilde{a}=\left(a_{1}, a_{2}, a_{3}\right)$, the graded mean representation of triangular fuzzy number $\tilde{a}$ is defined by (Kaufmann, A., \& Gupta, 1985):

$$
\operatorname{defuzz}(\tilde{a})=\frac{1}{6}\left(a_{1}+4 \times a_{2}+a_{3}\right) .
$$

\section{Proposed fuzzy VIKOR method}

This section focuses on a fuzzy VIKOR method in depth and provides a detailed analysis of its existing relations in finding the ideal solutions. In the previous studies, DM determines the relative importance of the positive ideal solution (PIS) and negative ideal solution (NIS) (i.e., value $v$ ). In some cases, DM may get confused in choosing the best suitable value for $v$. Moreover, failing to choose the proper value may change the ranking of alternatives, considerably. In this paper, new approach is thus introduced to solve this issue and the value is calculated in such a way that it does not depend on the DM. On the other hand, one of the main purposes of this section is the relationship among $R_{j}, S_{j}$ and $v$, which determines the value of $Q_{j}$. In fact, we concentrate on calculating the ranking parameter (i.e., ranking index $Q_{j}$ ) and the relation between $R_{j}$ and $S_{j}$ as given in traditional 
VIKOR method. Following describes an extended version of the VIKOR method under a fuzzy environment.

Based on the linear combination of the PIS and NIS in the fuzzy VIKOR method, alternatives are ranked according to the value of $Q_{j}$. In this combination, the relative importance of PIS and NIS (i.e., value $v$ ) is determined by the DM. The dependency of value $v$ to DM's preferences may affect on ranking alternatives. This is because of two main factors: 1) dissension within DMs and 2) failing to find relative importance of PIS and NIS properly. Therefore, in this paper, we determine the value of $v$, irrespective of the DMs.

The aim of extending the fuzzy VIKOR method is to determine the relative importance of distances as shown in Fig. 1 by $d^{+}$and $d^{-}$with respect to PIS and NIS. This method calculates the rectilinear distances from PIS to NIS. This is equivalent to the condition of the linear norm; therefore, a linear norm is utilized in order to get the relative importance of alternative(s) or point(s) with respect to PIS and NIS. In the fuzzy VIKOR method, a principle holds: the alternative with a shorter distance from PIS has the longer distance from NIS. Whilst in other compromise solution methods, such as the fuzzy TOPSIS method, in which distances are calculated based on the Euclidean-distance measure, the alternative with the shortest distance to PIS does not have the longest distance to NIS necessarily. This basic similarity between fuzzy VIKOR and linear norm is taken as driving force so that disutility weights of values $v$ and 1- $v$ in fuzzy VIKOR are calculated by the concept of linear norm and the locations of alternatives in $S-R$ space, as depicted in Fig. 1. This figure in fact shows the relation between PIS and NIS that is the square of unit scale. According to this figure, values of $v$ and $1-v$ for alternative $A_{j}$ can be computed by Eqs. (12-13).

$$
\begin{aligned}
& v=\frac{\|O A\|_{1}}{\|O B\|_{1}}=\frac{\left\|d_{j}^{+}\right\|}{\left\|d_{j}^{+}\right\|+\left\|d_{j}^{-}\right\|}=\frac{S_{j}+R_{j}}{2}, \\
& 1-v=\frac{\|A B\|_{1}}{\|O B\|_{1}}=\frac{\left\|d_{j}^{-}\right\|}{\left\|d_{j}^{+}\right\|+\left\|d_{j}^{-}\right\|}=\frac{2-\left(S_{j}+R_{j}\right)}{2},
\end{aligned}
$$

where,

$$
\begin{aligned}
& \|O A\|_{1}=\left\|d_{j}^{+}\right\|=S_{j}+R_{j}, \\
& \|A B\|_{1}=\left\|d_{j}^{-}\right\|=\left(1-S_{j}\right)+\left(1-R_{j}\right), \\
& \|O B\|_{1}=1+1=2 .
\end{aligned}
$$

By substituting Eq. (12) and Eq. (13) in the equation related to $Q_{j}$, the proposed ranking index can be obtained.

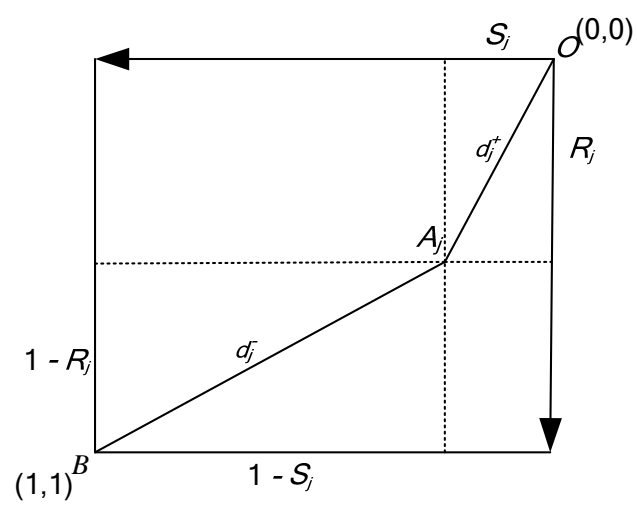

Fig. 1. Location of the alternative in $S-R$ space in the fuzzy VIKOR 
The main merit of this equation is that just by using the input data, the value of $Q_{j}$ is easily calculated without the need to ask the DM to determine the value of $v$ at the end of the fuzzy VIKOR process. The steps of the proposed VIKOR method under a fuzzy environment can be provided as follows:

Step 1: Choose the linguistic ratings $\left(\tilde{x}_{i j}, i=1,2, \ldots, m, j=1,2, \ldots, n\right)$ for alternatives with respect to criteria and the appropriate linguistic variables $\left(\tilde{w}_{j}, j=1,2, \ldots, n\right)$ for the weight of the criteria.

Step 2: If the supports of triangular fuzzy numbers do not belong to the interval $[0,1]$, then a scaling is needed to transform them back in this interval. Here, a linear scale transformation is used to have a comparable number.

$$
\tilde{f}_{i j}=\left(\frac{a_{i j 1}}{a_{i j 3}}, \frac{a_{i j 2}}{a_{i j 3}}, \frac{a_{i j 3}}{a_{i j 3}}\right) i=1,2, \ldots, m: j=1,2, \ldots, n
$$

where,

$$
\begin{aligned}
& \tilde{x}_{i j}=\left(a_{i j 1}, a_{i j 2}, a_{i j 3}\right) \\
& a_{i j 3}^{\max }=\max _{j} a_{i j 3}
\end{aligned}
$$

Step 3: Transform fuzzy decision matrix and fuzzy weighted value of each criterion into crisp values using the graded mean integration method by using Eq. (11).

Step 4: Determine the best $f_{j}^{*}$ and the worst $f_{j}^{-}$values of all criterion rating $(j=1,2, \ldots, n)$.

$$
\begin{aligned}
& f_{j}^{*}=\max _{i} f_{i j} \\
& f_{j}^{-}=\min _{i} f_{i j}
\end{aligned}
$$

Step 5: Compute the values $S_{i}$ and $R_{i}$ by:

$$
\begin{aligned}
& S_{j}=\sum_{j=1}^{n} w_{j}\left(f_{j}^{*}-f_{i j}\right) /\left(f_{j}^{*}-f_{j}^{-}\right), \\
& R_{j}=\max _{j} w_{j}\left(f_{j}^{*}-f_{i j}\right) /\left(f_{j}^{*}-f_{j}^{-}\right),
\end{aligned}
$$

Step 6: Compute the values by:

$$
Q_{j}=\frac{S_{j}+R_{j}}{2} \frac{\left(S_{j}-S^{*}\right)}{\left(S^{-}-S^{*}\right)}+\frac{2-\left(S_{j}+R_{j}\right)}{2} \frac{\left(R_{j}-R^{*}\right)}{\left(R^{-}-R^{*}\right)}
$$

where, $S^{*}=\min _{j} S_{j}, S^{-}=\max _{j} S_{j}, R^{*}=\min _{j} R_{j}, R^{-}=\max _{j} R_{j}$ and $v$ is introduced as a weight for the strategy of maximum group utility, whereas $1-v$ is the weight of the individual regret.

Step 7: Rank the alternatives, sorting by the values $S, R$, and $Q$ in decreasing order. The results are three ranking lists.

Step 8: Propose as a compromise solution the alternative $A^{(1)}$, which is the best ranked by the measure $Q$ (minimum) if the following two conditions are satisfied.

$\mathrm{C}_{1}$. Acceptance advantage: 
where, $A^{(2)}$ is the alternative with second position in the ranking list by $Q: D Q=1 / m-1$.

$C_{2}$. Acceptance stability in decision making:

Alternative $A^{(1)}$ must be also the best ranked by $S$ or/and $R$. This compromise solution is stable within a decision-making process. It can be the strategy of maximum group utility (when $v \geq 0.5$ is needed), or "by consensus" $v \approx 0.5$, or "with vector $(v \leq 0.5)$. Here, $v$ is the weight of decision-making strategy of maximum group utility.

If one of the condition is not satisfied, then a set of compromise solutions is proposed, which consists of:

- Alternatives $A^{(1)}$ and $A^{(2)}$ if only condition $\mathrm{C}_{2}$ is not satisfied, or

- Alternatives $A^{(1)}, A^{(2)}, \ldots, A^{(M)}$ if condition $C_{1}$ is not satisfied; $A^{(M)}$ is determined by the relation $Q\left(A^{(M)}\right)-Q\left(A^{(1)}\right) \prec D Q$, for maximum $M$ (the positions of these alternatives are "in closeness").

\section{Case Study}

This section considers the risk evaluating problem presented in Ebrahimnejad et al. (2010). It is a BOT large-scale project (i.e., power plant) in Iran. In the BOT project, the private sector builds and operates the power plant project at its own expense and in turn should receive the revenue from the project toll charge within some years, and then the project is transferred to the public sector. The detail of the risk management process for this large-scale project is not discussed in this paper; however, interested readers may refer to Ebrahimnejad et al. (2010) for more details of the BOT project. In this case study, the experiences of the private sector in the successful implementation of the BOT power plant project have been used. Understandably, the project management team would like to know whether the risks are important. The experience learned from this paper provides guidelines for the future project's risk evaluation problem, and analyzes potential risks in large-scale projects. We suggest some recommendations based on the obtained results for performance evaluation and a reference for future research.

Ebrahimnejad et al. (2010) considered project risk evaluation based on the fuzzy MCDM model. The first part of the model was the identification of risks that were effective on the activities explored during the life cycle of the BOT project. To examine and to identify risks in the project, final reports, historical information, questionnaires and other resourceful documents were used. In the second part of the model, risks with minor impacts and low probability of occurrences were eliminated from calculation and analysis. We extend the fuzzy MCDM model (i.e., proposed fuzzy VIKOR) and use the higher risks (i.e., ten risks) and selected criteria (i.e., five criteria) as fuzzy MCDM input data. Probability and impact criteria are not sufficient for covering all aspects of project risks alone. On the other hand, the fuzzy MCDM method gives an opportunity to take advantages of exact and appropriate criteria in order to increase the precision of final risks evaluation. Therefore, we utilize the following effective criteria based on developing risk concepts for more precise risk assessment (Ebrahimnejad et al., 2009; Ebrahimnejad et al., 2010; PMI, 2008).

1) Probability criterion: Risk probability assessment investigates the likelihood that each specific risk occurs.

2) Impact criterion: Risk impact assessment investigates potential effect on a project objective, such as time, cost, scope, or quality, including both negative effects for threats and positive effects for opportunities. 
3) Quickness of Reaction toward risk criterion: The duration of organization responses to the occurred event.

4) Event measure quantity criterion: Expected required resources for settling the event in an appropriate time and with standard equipment. In other words, this criterion indicates the cost price of the risk prevention.

5) Event capability criterion: The capability of the event falls into threats and opportunities outcomes.

We figure out that a triangular fuzzy number can adequately represent the fuzzy seven-scale linguistic variables, and it is thus used for the assessment hereafter (Ebrahimnejad et al., 2009; Ebrahimnejad et al., 2010). Table 1 illustrates the linguistic terms defined for the criteria of the project risk event.

\section{Table 1}

Relations between linguistic variables and triangular fuzzy numbers

\begin{tabular}{ll}
\hline Linguistic variables & Triangular fuzzy numbers \\
\hline very low (VL) & $(0,0,0.1)$ \\
low (L) & $(0,0.1,0.3)$ \\
medium low (ML) & $(0.1,0.3,0.5)$ \\
medium (M) & $(0.3,0.5,0.7)$ \\
medium high (MH) & $(0.5,0.7,0.9)$ \\
high (H) & $(0.7,0.9,1)$ \\
very high (VH) & $(0.9,1,1)$ \\
\hline
\end{tabular}

Table 2 illustrates the decision matrix that is the project expert's data derived by using DMs' judgment.

Table 2

Fuzzy decision matrix

\begin{tabular}{|c|c|c|c|c|c|}
\hline $\begin{array}{l}\text { Higher } \\
\text { risks }\end{array}$ & Description & $\begin{array}{l}\text { Probability } \\
\left(\mathrm{C}_{1}\right)\end{array}$ & $\begin{array}{l}\text { Quickness of reaction } \\
\text { toward risk } \\
\left(\mathrm{C}_{3}\right)\end{array}$ & $\begin{array}{l}\text { Event measure } \\
\text { quantity } \\
\left(\mathrm{C}_{4}\right)\end{array}$ & $\begin{array}{l}\text { Event capability } \\
\left(\mathrm{C}_{5}\right)\end{array}$ \\
\hline$\overline{A_{1}}$ & Financing & medium high & medium high & medium & high / medium high \\
\hline $\mathrm{A}_{2}$ & Delay or incompletion & amedium & medium high medium high & medium & medium high / high \\
\hline $\mathrm{A}_{3}$ & $\begin{array}{l}\text { Initial process } \\
\text { (technical and } \\
\text { financial studies) }\end{array}$ & high & medium & medium high & high / medium \\
\hline $\mathrm{A}_{4}$ & Procurement & medium high & medium high medium high & medium high & medium high / high \\
\hline $\mathrm{A}_{5}$ & Maintenance & medium low & medium low low & low & medium low / medium high \\
\hline $\mathrm{A}_{6}$ & Performance & medium & high & low & high / medium high \\
\hline $\mathrm{A}_{7}$ & Force major event & medium low & medium & medium & medium / medium \\
\hline$A_{8}$ & Expropriation & medium & medium high & high & medium / medium \\
\hline $\mathrm{A}_{9}$ & Breach of agreements & medium low & medium high medium & high & medium high / medium high \\
\hline$A_{10}$ & $\begin{array}{l}\text { Economics macro } \\
\text { factors } \\
\text { Weight }\end{array}$ & $\begin{array}{l}\text { medium high } \\
\text { high }\end{array}$ & $\begin{array}{l}\text { medium high medium low } \\
\text { very high medium high }\end{array}$ & $\begin{array}{l}\text { medium } \\
\text { medium high }\end{array}$ & $\begin{array}{l}\text { medium high / medium high } \\
\text { medium low }\end{array}$ \\
\hline
\end{tabular}

Fuzzy MCDM methods are powerful to solve complex problems raised in BOT projects to reach a reliable decision. A project risk evaluation model based on the TOPSIS as the rapid fuzzy MCDM and the LINMAP as the exact MCDM under a fuzzy environment were presented. Readers may refer to Ebrahimnejad et al. (2010) for details of the fuzzy TOPSIS and fuzzy LINMAP ranking processes for the ten higher alternatives.

We are motivated to continue the previous study in order to take the advantages of the fuzzy MCDM methods (i.e., fuzzy compromise solutions) as well as to present a new version of the fuzzy VIKOR method for modeling the project risk evaluation efficiently. Therefore, in this section besides two 
previous methods (i.e., TOPSIS and LINMAP), we introduce the VIKOR as the third well-known compromise solution method for the risk evaluation process under a fuzzy environment. The proposed fuzzy VIKOR method is an effective tool in MCDM, particularly in situations where the DM does not know how to express his/her preference at the beginning of the ranking process. The obtained compromise solution can be accepted by the DMs because it provides the maximum group utility of the "majority" (represented by min $S$, Eq. (17)), and the minimum individual regret of the “opponent”' (represented by min $R$, Eq. (18)). The compromise solutions can be the starting point for negotiation, involving the DMs' preference by criteria weights (Opricovic \& Tzeng, 2004, 2007). Moreover, in the fuzzy VIKOR both PIS and NIS are considered simultaneously. Hence, we explore the extended fuzzy VIKOR method in solving the proposed BOT project risk evaluation problem.

The linguistic weight of criteria and rating of alternatives with respect to criteria are converted into triangular fuzzy numbers using the seven-scale linguistic variables, as shown in Table 2. The values for the normalized decision matrix and the weight of each criterion are computed by Eq. (11) and Eq. (14). The best and worst values of all criterion ratings are determined using Eq. (15) and Eq. (16). Then, the values of $S, R$ and $Q$ are calculated for all higher risks. Table 3 illustrates the fuzzy VIKOR ranking of the risks by $S, R$ and $Q$ in a decreasing order.

Table 3

Values of $S, R$ and $Q$ for the higher risks

\begin{tabular}{lllll}
\hline Higher risks & $S$ & $R$ & $v$ & $Q$ \\
\hline $\mathrm{A}_{1}$ & 0.403 & 0.194 & 0.30 & 0.497 \\
$\mathrm{~A}_{2}$ & 0.602 & 0.194 & 0.40 & 0.607 \\
$\mathrm{~A}_{3}$ & 0.174 & 0.128 & 0.15 & 0.148 \\
$\mathrm{~A}_{4}$ & 0.467 & 0.194 & 0.33 & 0.526 \\
$\mathrm{~A}_{5}$ & 0.796 & 0.273 & 0.53 & 1.000 \\
$\mathrm{~A}_{6}$ & 0.390 & 0.194 & 0.29 & 0.493 \\
$\mathrm{~A}_{7}$ & 0.568 & 0.245 & 0.41 & 0.756 \\
$\mathrm{~A}_{8}$ & 0.581 & 0.194 & 0.39 & 0.592 \\
$\mathrm{~A}_{9}$ & 0.509 & 0.245 & 0.38 & 0.726 \\
$\mathrm{~A}_{10}$ & 0.371 & 0.097 & 0.23 & 0.073 \\
\hline
\end{tabular}

In this table, $v$ value is computed by Eq. (12) to show the decision-making strategy of the maximum group utility in order to compute $Q$ values. It is appropriate to mention that $v$ plays an important role in the ranking of risks (i.e., alternatives) (Büyüközkan, G., \& Ruan, 2008; Opricovic, S., \& Tzeng, 2004, 2007). In this section, the proposed ranking indices for $Q_{j}$ are applied to solve the dependency problem to the DM. Ranking of ten higher risks in the case study based on the extended fuzzy VIKOR method are shown (See Table 3). Moreover, a sensitivity analysis on $v$ taking in the interval $[0,1]$ is conducted. The related results are illustrated in Table 4.

\section{Discussion on case study}

In this section, a comparison is made to show advantages and disadvantages of the proposed fuzzy VIKOR, fuzzy TOPSIS and fuzzy LINMAP methods. We illustrate the computational results of three well-known fuzzy compromise solution methods in the project risk evaluation. The results obtained from the fuzzy LINMAP are different from the fuzzy VIKOR and fuzzy TOPSIS methods as illustrated in Table 5. The main reason of this type of tangible difference between resulting ranks of the fuzzy LINMAP and the other two methods is on the accuracy of the pair-wise comparison of alternatives. In fact, when the number of alternatives increases the number of pairwise comparison increases too and this makes it difficult for use traditional LINMAP. As can be seen from Tables 4 
and 5, the final ranks of the proposed fuzzy VIKOR and fuzzy TOPSIS, with high value of $v$, are similar. This similarity can be regarded as a proof for accuracy and stability of final ranks resulted from these two fuzzy MCDM methods.

\section{Table 4}

Risk ranking for different values of $v$

\begin{tabular}{lllllll}
\hline & & \multicolumn{5}{c}{ Fuzzy VIKOR } \\
\cline { 3 - 7 } Higher risks & Description & 0 & 0.3 & 0.5 & 0.7 & 1 \\
\hline $\mathrm{A}_{1}$ & Financing & $\mathrm{A}_{10}$ & $\mathrm{~A}_{10}$ & $\mathrm{~A}_{3}$ & $\mathrm{~A}_{3}$ & $\mathrm{~A}_{3}$ \\
$\mathrm{~A}_{2}$ & Delay or incompletion & $\mathrm{A}_{3}$ & $\mathrm{~A}_{3}$ & $\mathrm{~A}_{10}$ & $\mathrm{~A}_{10}$ & $\mathrm{~A}_{10}$ \\
$\mathrm{~A}_{3}$ & Initial process (technical and financial studies) & $\mathrm{A}_{6}$ & $\mathrm{~A}_{6}$ & $\mathrm{~A}_{6}$ & $\mathrm{~A}_{6}$ & $\mathrm{~A}_{6}$ \\
$\mathrm{~A}_{4}$ & Procurement & $\mathrm{A}_{1}$ & $\mathrm{~A}_{1}$ & $\mathrm{~A}_{1}$ & $\mathrm{~A}_{1}$ & $\mathrm{~A}_{1}$ \\
$\mathrm{~A}_{5}$ & Maintenance & $\mathrm{A}_{2}$ & $\mathrm{~A}_{4}$ & $\mathrm{~A}_{4}$ & $\mathrm{~A}_{4}$ & $\mathrm{~A}_{4}$ \\
$\mathrm{~A}_{6}$ & Performance & $\mathrm{A}_{4}$ & $\mathrm{~A}_{8}$ & $\mathrm{~A}_{8}$ & $\mathrm{~A}_{8}$ & $\mathrm{~A}_{9}$ \\
$\mathrm{~A}_{7}$ & Force major event & $\mathrm{A}_{8}$ & $\mathrm{~A}_{2}$ & $\mathrm{~A}_{2}$ & $\mathrm{~A}_{9}$ & $\mathrm{~A}_{7}$ \\
$\mathrm{~A}_{8}$ & Expropriation & $\mathrm{A}_{7}$ & $\mathrm{~A}_{9}$ & $\mathrm{~A}_{9}$ & $\mathrm{~A}_{2}$ & $\mathrm{~A}_{8}$ \\
\hline $\mathrm{A}_{9}$ & Breach of agreements & $\mathrm{A}_{9}$ & $\mathrm{~A}_{7}$ & $\mathrm{~A}_{7}$ & $\mathrm{~A}_{7}$ & $\mathrm{~A}_{2}$ \\
$\mathrm{~A}_{10}$ & Economics macro factors & $\mathrm{A}_{5}$ & $\mathrm{~A}_{5}$ & $\mathrm{~A}_{5}$ & $\mathrm{~A}_{5}$ & $\mathrm{~A}_{5}$ \\
\hline
\end{tabular}

For the extended fuzzy VIKOR method, it is worth noting that the method' weights are determined by the disutility of PIS and NIS. This extended method, which is based on a linear norm in $R^{2}$ and the location of alternatives $\left(S_{j}, R_{j} \in[0,1]\right.$ ), calculates the value of $v$ for each alternative, separately. This is the superiority of this method, so the ranking of each alternative is determined based on its location in the $[0,1] \times[0,1]$ space.

\section{Table 5}

Three fuzzy compromise solution methods

\begin{tabular}{lllll}
\hline \multirow{2}{*}{ Higher risks } & Description & $\begin{array}{l}\text { Proposed fuzzy } \\
\text { VIKOR ranking }\end{array}$ & $\begin{array}{l}\text { Fuzzy TOPSIS } \\
\text { ranking }\end{array}$ & $\begin{array}{l}\text { Fuzzy LINMAP } \\
\text { ranking }\end{array}$ \\
\hline $\mathrm{A}_{1}$ & Financing & $\mathrm{A}_{10}$ & $\mathrm{~A}_{3}$ & $\mathrm{~A}_{3}$ \\
$\mathrm{~A}_{2}$ & Delay or incompletion & $\mathrm{A}_{3}$ & $\mathrm{~A}_{10}$ & $\mathrm{~A}_{10}$ \\
$\mathrm{~A}_{3}$ & $\begin{array}{l}\text { Initial process } \\
\text { (Technical and financial studies) }\end{array}$ & $\mathrm{A}_{6}$ & $\mathrm{~A}_{1}$ & $\mathrm{~A}_{2}$ \\
$\mathrm{~A}_{4}$ & Procurement & $\mathrm{A}_{1}$ & $\mathrm{~A}_{6}$ & $\mathrm{~A}_{9}$ \\
$\mathrm{~A}_{5}$ & Maintenance & $\mathrm{A}_{4}$ & $\mathrm{~A}_{4}$ & $\mathrm{~A}_{8}$ \\
$\mathrm{~A}_{6}$ & Performance & $\mathrm{A}_{8}$ & $\mathrm{~A}_{9}$ & $\mathrm{~A}_{1}$ \\
$\mathrm{~A}_{7}$ & Force major event & $\mathrm{A}_{2}$ & $\mathrm{~A}_{8}$ & $\mathrm{~A}_{7}$ \\
$\mathrm{~A}_{8}$ & Expropriation & $\mathrm{A}_{9}$ & $\mathrm{~A}_{7}$ & $\mathrm{~A}_{4}$ \\
$\mathrm{~A}_{9}$ & Breach of agreements & $\mathrm{A}_{7}$ & $\mathrm{~A}_{2}$ & $\mathrm{~A}_{6}$ \\
$\mathrm{~A}_{10}$ & Economics macro factors & $\mathrm{A}_{5}$ & $\mathrm{~A}_{5}$ & $\mathrm{~A}_{5}$ \\
\hline
\end{tabular}

Finally, in terms of the accuracy of the proposed model, the computational results show that the fuzzy TOPSIS method finds the best possible risk (i.e., alternative) among the gathered and existing ranking data, while the fuzzy LINMAP method finds the optimal solution based on the exact method by considering all risk evaluation data. In the fuzzy LINMAP method, the best alternative is the one with the least distance from the optimal point. Furthermore, the fuzzy LINMAP method is based on pair-wise comparisons of alternatives provided by DM and generates the best compromise alternative(s) as the solution with the shortest distance to the PIS. The proposed fuzzy VIKOR method, like fuzzy TOPSIS, finds the best possible alternative(s) considering both positive and 
negative ideal solutions. However, the most important point is that the fuzzy VIKOR compared to fuzzy TOPSIS considers the relative distance to both PIS and NIS, simultaneously.

The decision-making process in project management deals with identification, classification and selection problems. Indeed, project risk management is one of the fields where decision-making methods play an important role. Risk evaluation is the main phase in project risk management process. An increase in long lasting complicated processes and organizational features is expected, which result in large-scale projects with high risks. Large-scale projects often result in cost overrun, schedule delays because high risks are not evaluated, properly. Setting priority the high risks for these projects is a complex task since there is generally not enough information available to analyze risks and their impacts on the project or organization. Hence, strategic decisions have to be made with these levels of uncertainties. There is a limit to the resources (e.g., labor forces or human, financial and technical aspects) that can be made available for project managers. On the other hand, the risk evaluation is vital in order to mitigate their impacts and to measure risk effect based on predetermined criteria. It can provide some approaches to cope with these risks in a sub-sequent phase of the risk management process.

\section{Conclusion}

In this paper, five criteria have been applied as risk evaluation criteria, unlike many previous studies. A new approach presented for the risk evaluation to deal with risks associated with large-scale projects in the complicated situations, in which the information to analyze risks is unquantifiable, incomplete or non-obtainable. The proposed approach based on the extended VIKOR method helps the experts in the risk management team make their judgments by using linguistic terms instead of real numbers. Since linguistic terms are not mathematically operable, to solve this difficulty, each linguistic term is transformed into a triangular fuzzy number, which describes the meaning of each verbal term. The fuzzy VIKOR method has been extended to provide the DMs with appropriate tool for decision-making purposes. In the traditional VIKOR method, the DM has to select a value for parameter $v$. Failing to choose a proper value for it may change the output and alternative ranking, considerably. Therefore, we have presented the extended fuzzy VIKOR method, which can determine the value of parameter $v$ without having additional information. The risk evaluation problem is often influenced by uncertainty in large-scale projects. In such situation, the fuzzy set theory is an appropriate tool to deal with this kind of problems. In real-life decision-making process, the DM cannot express his preferences precisely in numerical values, and the evaluations are often expressed in linguistic terms. Thus, in this paper, an MCDM approach under a fuzzy environment has been proposed to handle both qualitative and quantitative criteria, and analyze the high risks, effectively. Moreover, an application of the MCDM in the field of project risk evaluation has been thoroughly studied. Through a real case in the power industry project, the usability of well-known MCDM methods, including the proposed fuzzy VIKOR and other two methods, namely fuzzy TOPSIS and fuzzy LINMAP, is conducted. Furthermore, the advantages of the proposed fuzzy VIKOR method along with other two powerful MCDM are discussed.

\section{Acknowledgment}

The first author is grateful for the partially financial support from the Islamic Azad University - Karaj Branch under the research project (No. 1/73295). The authors also wish to thank the power industry experts for their contributions on this research.

\section{References}

Al-Subhi Al-Harbi, K.M. (2001). Application of the AHP in project management, International Journal of Project Managements, 19, 19-27. 
Büyüközkan, G., \& Ruan, D. (2008). Evaluation of software development projects using a fuzzy multi-criteria decision approach, Mathematics and Computers in Simulation, 77, 464-475.

Chen, L.Y., \& Wang, T.-C. (2009). Optimizing partners' choice in IS/IT outsourcing projects: The strategic decision of fuzzy VIKOR, International Journal of Production Economics, 120, 233-242.

Chin, K., Xu, D., Yang, J., \& Lam, J. (2008). Group-based ER-AHP system for product project screening, Expert System with Application, 35(4). 1909-1929.

Dey, P.K. (2002). Project risk management: a combined analytic hierarchy process and decision tree analysis approach, Cost Engineering Journal, 44 (3). 13-26.

Dey, P.K., \& Ogunlana, S.O. (2004). Selection and application of risk management tools and techniques for build-operate-transfer project, Industrial Management and Data System, 104(4). 334-346.

Dey, P.K., Tabucanon, M.T., \& Ogunlana, S.O. (1994). Planning for project control through risk analysis; a case of petroleum pipeline laying project, International Journal of Project Management, 12(1). 23-33.

Ebrahimnejad, S., Mousavi, S.M., \& Mojtahedi, S.M.H. (2008). A model for risk evaluation in construction projects based on fuzzy MADM, In: Proceeding of the 4th IEEE International Conferences on Management of Innovation \& Technology, Thailand, 305-310.

Ebrahimnejad, S., Mousavi, S.M., \& Mojtahedi, S.M.H. (2009). A fuzzy decision making model for risk ranking with application to the onshore gas refinery, International Journal of Business Continuity and Risk Management, 1, 38-66.

Ebrahimnejad, S., Mousavi, S.M., \& Seyrafianpour, H. (2010). Risk identification and assessment for build-operate-transfer projects: A fuzzy multi attribute decision making model, Expert System with Application, 37, 575-586.

Kahraman, C., Büyüközkan, G., \& Yasin Ates, N. (2007). A two phase multi-attribute decisionmaking approach for new product introduction, Information Sciences, 177, 1567-1582.

Kaufmann, A., \& Gupta, M.M. (1985). Introduction to fuzzy arithmetic: theory and applications, Van Nostrand Reinhold, New York.

Kumar Dey, P. (2010). Managing project risk using combined analytic hierarchy process and risk map, Applied Soft Computing, 10, 990-1000.

Li, D., \& Yang, J.B. (2004). Fuzzy linear programming technique for multi-attribute group decision making in fuzzy environments, Information Sciences, 158, 263-275.

Makui, A., Mojtahedi, S.M.H., \& Mousavi, S.M. (2010). Project risk identification and analysis based on group decision making methodology in a fuzzy environment, International Journal of Management Science and Engineering Management, 5(2). 108-118.

Mojtahedi, S.M.H., Mousavi, S.M., \& Makui, A. (2010). Project risk identification and assessment simultaneously using multi-attribute group decision making technique, Safety Science, 48(4). 499507.

Mousavi, S.M., Tavakkoli-Moghaddam, R., Azaron, A., Mojtahedi, S.M.H., \& Hashemi, H. (2011). Risk assessment for highway projects using jackknife technique, Expert Systems With Applications, 38, 5514-5524.

Mousavi, S.M., Tavakkoli-Moghaddam, R., Hashemi, H., \& Mojtahedi, S.M.H. (2011b). A novel approach based on non-parametric resampling with the interval analysis for large engineering project risks. Safety Science, 49(10), 1340-1348.

Mustafa, M.A., \& Al-Bahar, J.F. (1991). Project risk assessment using the analytic hierarchy process, IEEE Transactions on Engineering Management, 38(1). 46-52.

Nieto-Morote, A., \& Ruz-Vila, F. (2011). A fuzzy approach to construction project risk assessment, International Journal of Project Management, 29, 220-231.

Opricovic, S. (1998). Multicriteria optimization of civil engineering systems, Faculty of Civil Engineering, Belgrade.

Opricovic, S., \& Tzeng, G.-H. (2004). Compromise solution by MCDM methods: A comparative analysis of VIKOR and TOPSIS. European Journal of Operational Research, 156, 445-455. 
Opricovic, S., \& Tzeng, G.-H. (2007). Extended VIKOR method in comparison with outranking methods, European Journal of Operational Research, 178, 514-529.

PMI. (2008). A guide to the project management body of knowledge (PMBOK Guide). 4th ed., USA, Project Management Institute Inc., Chapter 11 on project risk management.

Tavakkoli-Moghaddam, R., Mousavi, S.M., \& Hashemi, H. (2011). A fuzzy comprehensive approach for risk identification and prioritization simultaneously in EPC projects, InTech Publisher, Published in the book "Risk Management / Book 2", ISBN 978-953-307-482-5.

Tavakkoli-Moghaddam, R., Mousavi, S.M., \& Heydar, M. (2011). An integrated AHP-VIKOR methodology for plant location selection, International Journal of Engineering, Article in press.

Tsuar, S.H., Chang, T.Y., \& Yen, C.H. (2002). Evaluation of airline service quality by fuzzy MCDM, Tourism Management, 23, 107-115.

Wang, T.-C., \& Chang, T.-H. (2007). Application of TOPSIS in evaluating initial training aircraft under a fuzzy environment, Expert System with Application, 33, 870-880.

Wu, H.-Y., Tzeng, G.-H., \& Chen, Y.-H. (2009). A fuzzy MCDM approach for evaluating banking performance based on Balanced Scorecard, Expert System with Application, 36, 10135-10147.

Xia, H.C., Li, D.F., Zhou, J.Y., \& Wang, J.M. (2006). Fuzzy LINMAP method for multi attribute decision making under fuzzy environments, Journal of Computer and System Sciences, 72, 741759.

Zayed, T., Amer, M., \& Pan, J. (2008). Assessing risk and uncertainty inherent in Chinese highway projects using AHP, International Journal of Project Management, 26, 406-419.

Zeng, J., An, M., \& Smith, N.J. (2007). Application of a fuzzy based decision making methodology to construction project risk assessment, International Journal of Project Management, 25, 589600.

Zimmermann, H.J. (1991). Fuzzy set theory and its applications, 2nd ed., Boston, Dordrecht, London: Kluwer Academic Publishers. 